\title{
Concepts and Indexes of Elder Abuse: The Conceptual Framework for Applied Studies in the Field of Elder Abuse
}

Fatemeh Estebsari

* PhD in Health Education \& Health Promotion, Assistant Professor, Dept. of Community Health Nursing, School of Nursing \& Midwifery, Shahid Beheshti Univ. of Medical Sciences (SBMU), Tehran, Iran. (Corresponding Author) fa_estebsari@yahoo.com

Davood Mostafaei

$\mathrm{PhD}$ in Healthcare Management, Assistant Professor, Dept. of Nursing Management, School of Nursing \& Midwifery, SBMU, Tehran, Iran.

Zahra Rahimi Khalifehkandi MSc Student in Health Education \& Health Promotion, Dept. of Health Education \& Health Promotion, School of Health, Iran Univ. of Medical Sciences (IUMS), Tehran, Iran.

Kimia Estebsri

BSc in Midwifery, Maternal Health Expert, Family Health Unit, Azna Health Network, Azna Health Center, Lorestan University of Medical Sciences (LUMS), Lorestan, Iran.

Mohammad Hossain Taghdisi Full Professor, Dept. of Health Education \& Health Promotion, School of Health, Iran Univ. of Medical Sciences (IUMS), Tehran, Iran.

Received: 29 May 2016 Accepted: 01 November 2016 DOI: 10.18869/acadpub.ihepsaj.5.1.15

\section{ABSTRACT}

Background and Objective: Due to physiological and anatomical changes elders are more susceptible and exposed to numerous dangers including elder abuse. Elder abuse is a complex issue; so that it is mentioned as one of the indirect predictors of death which it is hard to assess. The objective of this study was to make researchers become familiar with the concepts, types and indexes of elder abuse. To reach a uniform concept towards this issue will help to make more cohesive actions to track and reduce cases of elder abuse.

Results: Different definitions of elder abuse and a variety of elder abuse, indicators and behaviors associated with each type of harassment was offered. The first and most important step in dealing with a phenomenon is to become familiar with the concepts and terminology position.

Conclusion: Based on the findings of this study, uniform concepts in the field of elder abuse and its different variety will help policy makers and planners have access to the uniform concept in this field. In addition, it is used in setting policy and related policies for the elderly to pay special attention to elder abuse as a specialized subject.

Keywords: Elders, Elderly, Elder abuse, Index of Elder Abuse, Domestic violence. Paper Type: Review Article.

- Citation (Vancouver): Estebsari F, Mostafaei D, Rahimi Z, Estebsri K, Taghdisi $\mathrm{MH}$. Concepts and indexes of elder abuse: the conceptual framework for applied studies in the field of elder abuse. Iran J Health Educ Health Promot. Spring 2017;5(1):15-19. [Persian]

- Citation (APA): Estebsari, F, Mostafaei, D, Rahimi, Z, Estebsri, K, Taghdisi MH. (Spring 2017). Concepts and indexes of elder abuse: the conceptual framework for applied studies in the field of elder abuse. Iranian Journal of Health Education \& Health Promotion., 5(1),15-19. [Persian] 


\title{
مفاهيم و شاخصهاى سالمندآزارى: خارجوب مفهومى براى مطالعات كاربردى
}

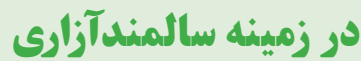

\section{-}

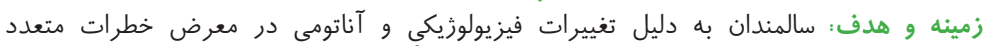

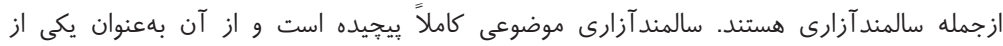

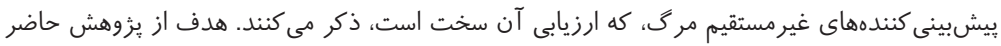

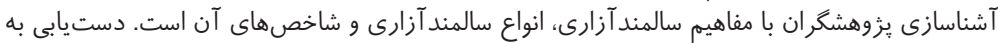

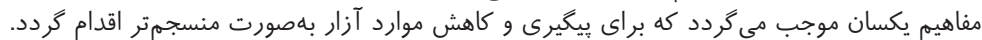

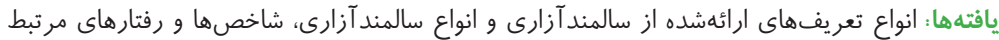

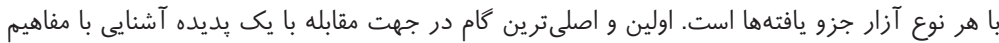

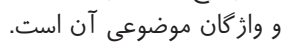

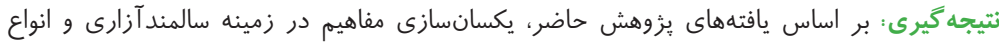

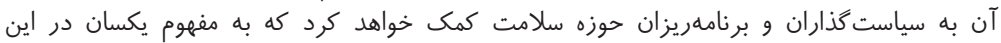

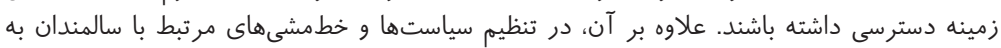

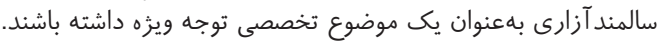

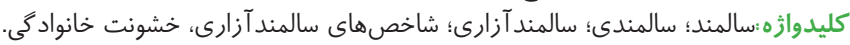
نوع مقاله: مطالعه مرورى

4 استناد (ونكوور): استبصارى ف، مصطفايى د، رحيمى ز، استبصارى ك، تقديسى محار مارى مفاهيم و

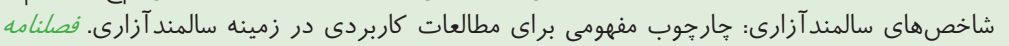

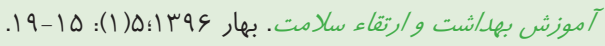

4 استناد (APA): استبصارى، فاطمه؛ مصطفايى، داود؛ رحيمى، زهرا؛ استبصارى، كيميا؛ تقديسى،

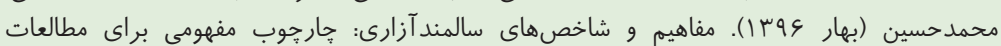

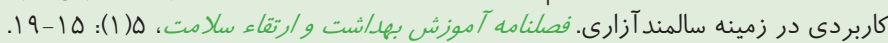

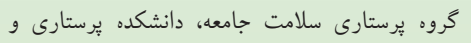

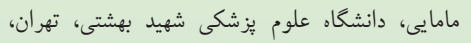

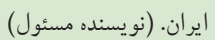
fa_estebsari@yahoo.com

داود مصطفايى دكتراى مديريت خدمات بهداشتى درمانى، استاديار

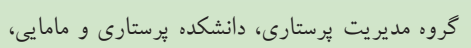
دانشكاء علوم يزشكى شهيد بهشتى، تهران، ايران.

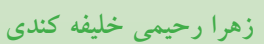

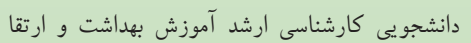

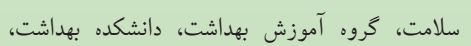
دانشكاه علوم يزشكى ايران، ايران.

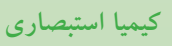
كارشناس مامايى، كارشناس سلامت مارئ مادران، شبكه

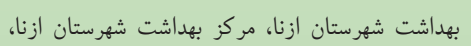

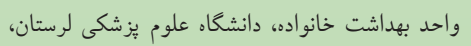

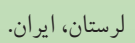

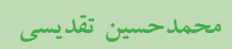
استاد تمام كروه خدمات بهداشتى و آموزش بهدئ بهداشت،

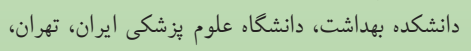




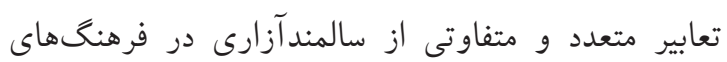

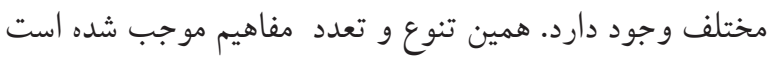

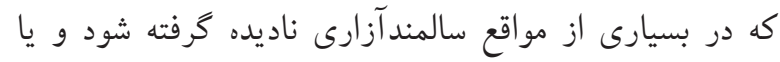

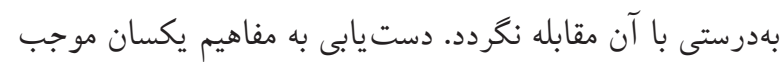

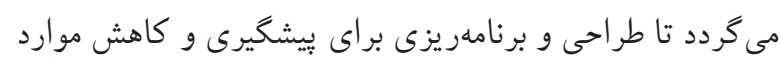

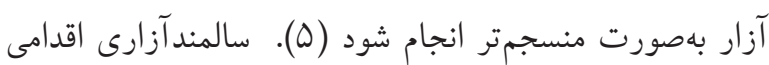

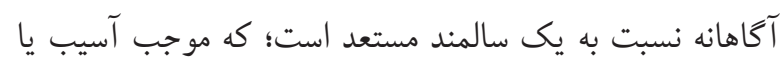

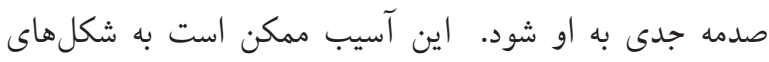
مختلف باشد: آكاهانه و توسط مراقبت كننده يا فردى كه مودي الئ مورد اعتماد سالمند بوده و داراى رابطه قابلاعتماد با اوست انجام شود؛ يا ممكن است ناشى از نارسايى و كوتاهى خود فرد در رفع نيازهاى اوليه و محافظت از خود در برابر صدمات باشد آداند

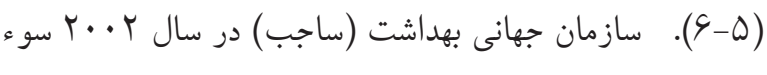
رفتار را بهعنوان يك اقدام منفرد يا تكرارشونده يا عدم اقدام

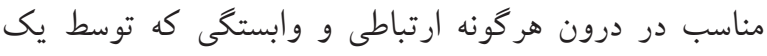

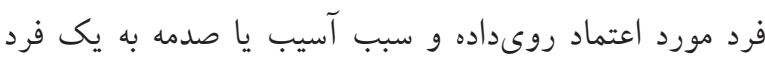

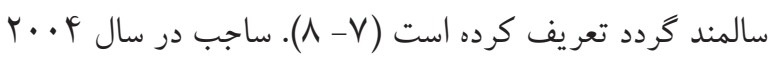

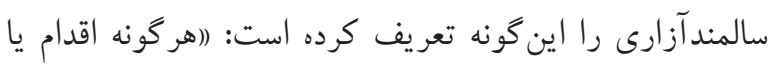
عدم اقدام است كه نتيجهاش آسيب به يك فرد سالمند شود (ه). استفاده از عبارت ((هر نوع آسيبى) و (امىتو اند شامل شود)) اين امكان را فراهم مى كند كه دامنه وسيعى از تعريف و تفسيرها

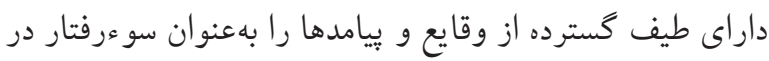

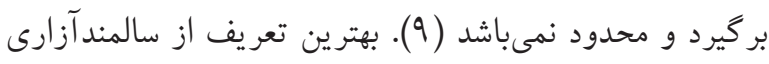

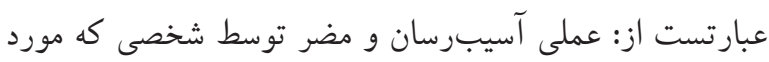

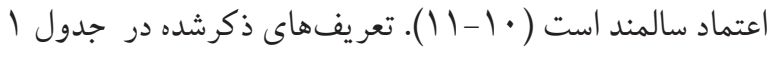

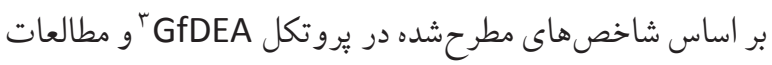
مرورى انجامشده است.

3. Protocols GfDEA Guidelines for Developing Elder Abuse)
يكى از بارزترين تغييرات جمعيتشناختى قرن بيستويكم

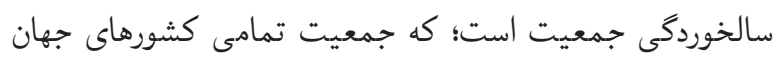

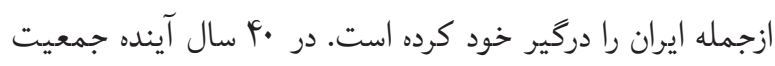

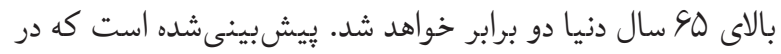

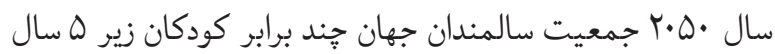

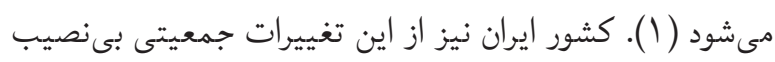

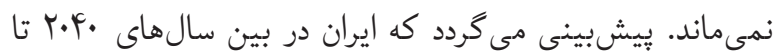

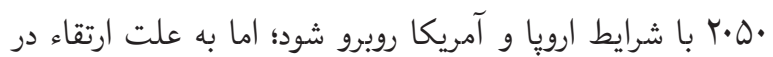

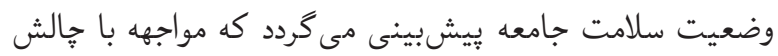
سالمندى در كشور ما حداقل يك دهه زودتر اتفاق بيافتد(Y). سالمندان به دليل تغييرات فيزيولوزيكى و آناتومى ناشى ازئ داز افزايش سن زيستى، بازنشستخى و كاهش فعاليتهاى اجتماعى، مرگ خويشاوندان و دوستان، دورى فرزندان به دليل ازدواج، كار يا

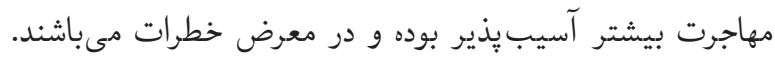
يكى از عوامل خطر براى سالمندان، سالمندآزارى' است.

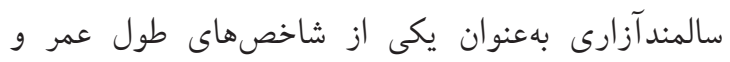
سلامت، در كنار شاخصهايى مثل اميد زندگى است (T).

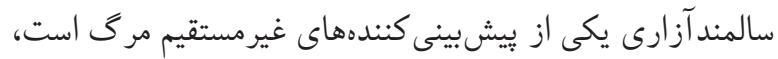

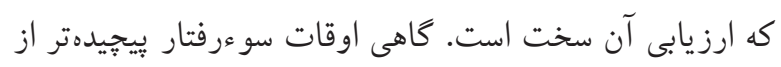

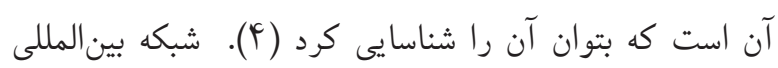

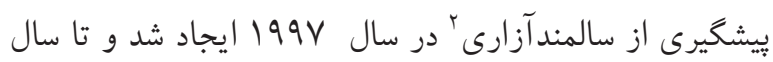

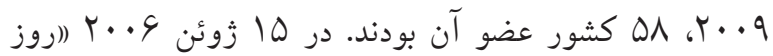

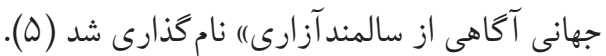

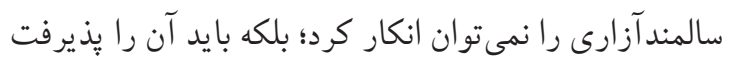

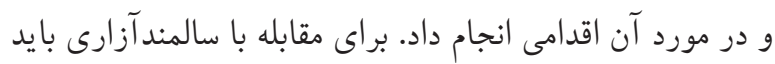

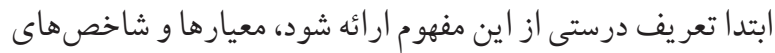

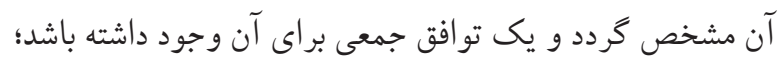

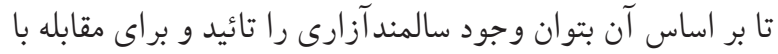

1. Elder abuse

2. International Network Prevention of Elder Abuse (INPEA) 
جدول ا. تعريف، شاخصها و رفتارهاى مرتبط با انواع سالمندآزارى

\begin{tabular}{|c|c|c|c|}
\hline رفتارها & شاخصها & تعريف & انواع سالمندآزارى \\
\hline 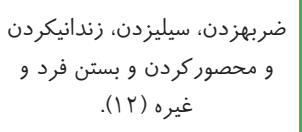 & | & 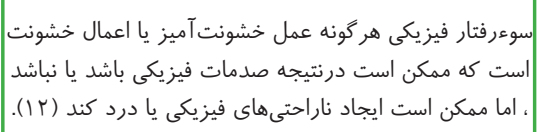 & فيزيكى' \\
\hline توهين و و اهانت و سرزيى، كنار گذاشتن تحقير از & 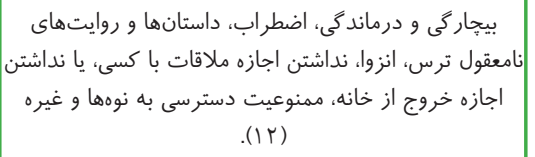 & 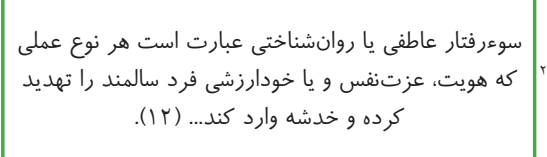 & عاطفى يا روانشناختى' \\
\hline 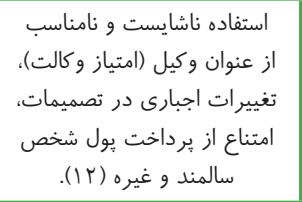 & 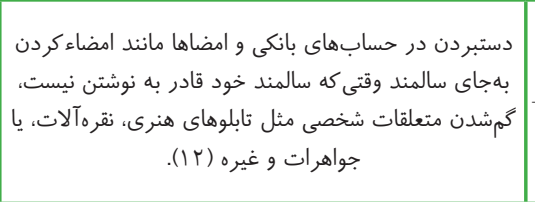 & 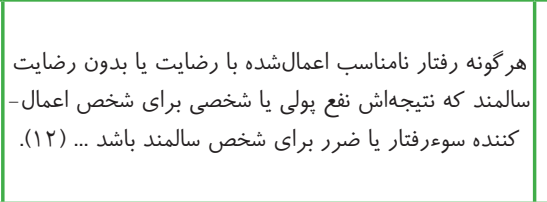 & 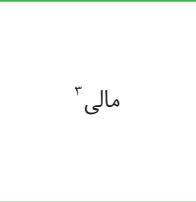 \\
\hline 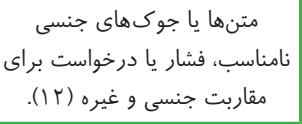 & 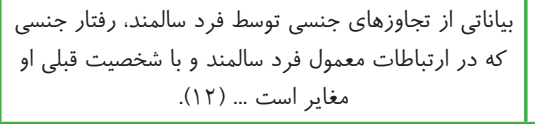 & 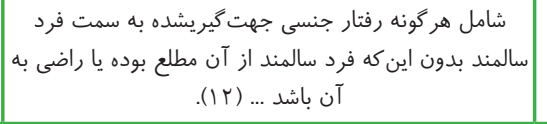 & جنسى" \\
\hline \multirow[t]{6}{*}{ نار سايى در تهيه غذا، مسكن، } & 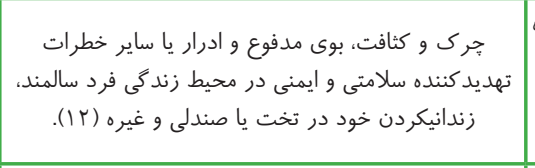 & 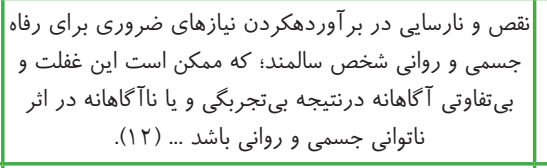 & غفلت \\
\hline & 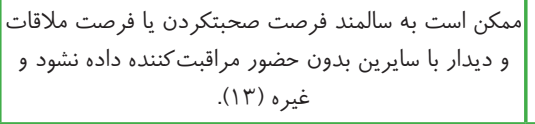 & 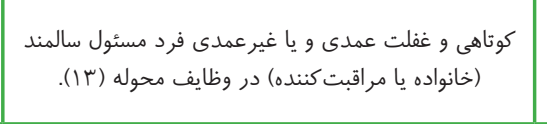 & غفلت مراقبت كننده \\
\hline & 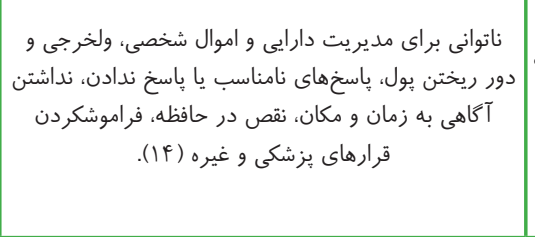 & 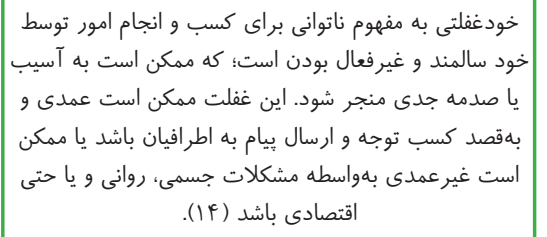 & خودغفلتى" \\
\hline & 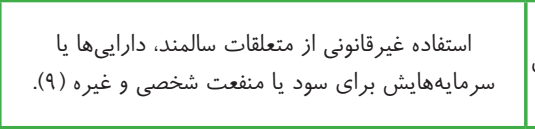 & 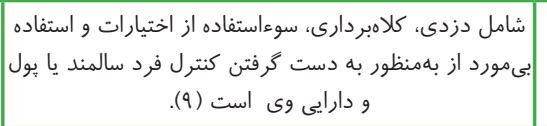 & استثمار \\
\hline & 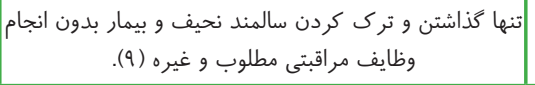 & ترى كردن شخص و تنها گذاشتن وى بدون يِيخيرى (9). & ترى و رهاسازى \\
\hline & & 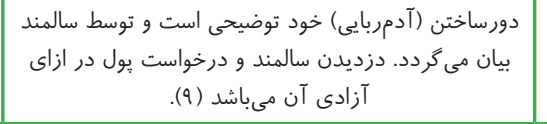 & آدمربايى' \\
\hline 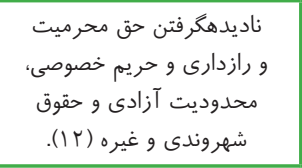 & 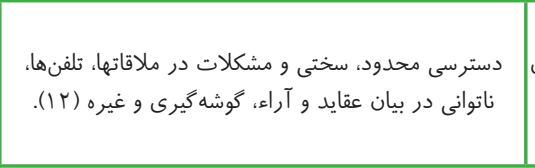 & 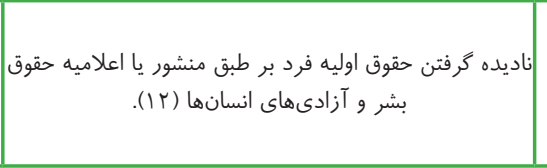 & ناديده گرفتن حقوق \\
\hline - & - & 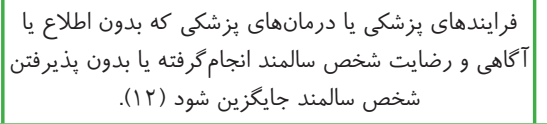 & سوء رفتار يزشكى \\
\hline
\end{tabular}

1. physical abuse

3. financial abuse

5. neglect

7. Abduction
2. psychological abuse

4. sexual abuse

6. self-neglect 
موقعيتهاى گوناگون تائيد شده باشد (1V-1V). تاكنون جندين ابزارها با قابليت اجرايى طراحىشده است. ابزارهاى غربالكرى

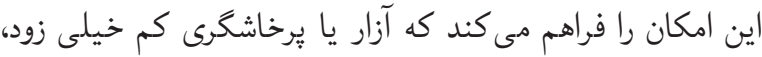

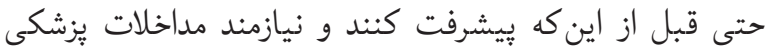

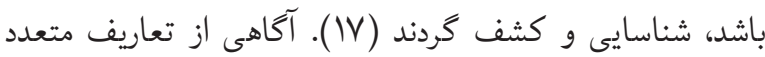

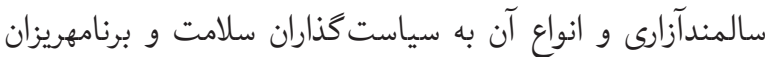
سلامتى كمك خواهد كرد تا در سياست گذارى و خطمشىهاى خود به موضوع سالمندآزارى, بهعنوان يك موضوع تخصصى، توجه

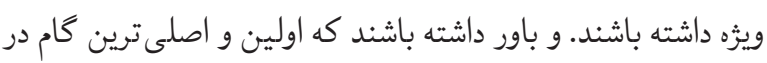
جهت مقابله با خطر سالمندآزارى، آشنايى با مفاهيم سالمندآزارى باري

\section{References:}

$$
\text { و شاخصهاى آن است. }
$$

1. Dadkhah a. Aging care system in America and Japan and to present indicators for strategic planning at Iran's aging services. Iranian Jornal of Ageing 2007;2(3):166-76.

2. Haghighatian M, Fotouhi M. Sociocultural Factors Affecting Elderly Abuse. J Health System Res. 2013;8(7):1117-26. http://hsr.mui.ac.ir/index.php/jhsr/article/view/298.

3. Fraga S, Lindert J, Barros $H$, Torres-Gonzalez F, loannidiKapolou E, Melchiorre MG, et al. Elder abuse and socioeconomic inequalities: A multilevel study in 7 European countries. Preventive Medicine. 2014:. http:// dx.doi.org/10.1016/j.ypmed.2014.01.008.

4. Strasser S, King P, Payne B, O'Quin K. Elder Abuse: What Coroners Know and Need to Know. ournal of Elder Abuse \& Neglect. 2013;25(3):242-53. DOI:10.1080/08946566.20 12.751826.

5. Biggs S, Haapala I. Theoretical Development and Elder Mistreatment:Spreading Awareness and Conceptual Complexity in Examining the Management of SocioEmotional Boundaries. Ageing Int. 2010;35:171-84.

6. Lee YS, Moon A, Gomez C. Elder mistreatment, culture, and help-seeking: a cross-cultural comparison of older Chinese and Korean immigrants. J Elder Abuse Negl. 2014;26(3):244-69.

7. Simone L, Wettstein A, Senn O, Rosemann T, Hasler S. Types of abuse and risk factors associated with elder abuse. Swiss medical weekly. 2016;146:w14273.PMID: 26827053

8. Sandmoe A, Kirkevold M. Identifying and handling abused older clients in community care: the perspectives of nurse managers. International journal of older people nursing. 2013;8(2):83-92.

9. Johannesen $M$, LoGiudice D. Elder abuse: a systematic review of risk factors in community-dwelling elders. Age

$$
\text { بحث و نتيجه كيرى }
$$

سالمندآزارى موضوعى يِيجيده است و تشخيص سالمندآزارى

نيازمند به دستيابى به تعريف يكسان، واضح و مشخص از سالمندآزارى موردقبول مراجع ذىصلاح و متناسب با فرهنگ به جامعه است (10). بهطورى كه بتوان بر اساس شاخصهاى رفتارى بهطور يقين نوع آزار و ميزان آن را تشخيص داد. حتى بتوان افراد در معرض خطر سالمندآزارى را قبل از اين كه فاجعهاى به بار آورد را شناسايى كرد. در اين راستا، اهميت وجود ابزارهاى غربالكرى و ارزيابى سالمندآزارى، كه داراى قابليت اجرايى باشند, آشكار مى گردد. ابزارهايى كه از جانب متخصصان مراقبت از سلامتى قابليت اعتماد و مورد تائيد باشند و روايى و ياياى آنها در

Ageing. 2013;42(3):292-8.

10. Strasser SM, Kerr J, King PS, Payne B, Beddington S, Pendrick $D$, et al. A survey of georgia adult protective service staff: implications for older adult injury prevention and policy. West J Emerg Med. 2011;12(3):357-64.

11. Jargin SV. Elder abuse and neglect versus parricide. International journal of high risk behaviors \& addiction. 2013;2(3):136-8.

12. Protocols GfDEA. Guidelines for Developing Elder Abuse Protocols,A South West Ontario Approach,February 22, 2011Approved by South West Regional Elder Abuse Network. 2011.

13. Dong X, Simon M, Evans D. Elder Self-Neglect Is Associated With Increased Risk for Elder Abuse in a CommunityDwelling Population Findings From the Chicago Health and Aging Project. J Aging Health 2013;25(1):180-96. doi: 10.1177/0898264312467373.

14. Martins R, Neto MJ, Andrade A, Albuquerque C. Abuse and maltreatment in the elderly. Atencion primaria / Sociedad Espanola de Medicina de Familia y Comunitaria. 2014;46 Suppl 5:206-9. 10.1016/s0212-6567(14)70093-9. PMID: 25476063.

15. Cherry KE, Brown JS, Kim S, Jazwinski SM. Social Factors and Healthy Aging: Findings from the Louisiana Healthy Aging Study (LHAS). Kinesiology review (Champaign, III). 2016;5(1):50-6.

16. Strasser SM, Smith M, Weaver S, Zheng S, Cao Y. Screening for Elder Mistreatment among Older Adults Seeking Legal Assistance Services. West J Emerg Med. 2013;14(4):309-15.

17. O'Brien JG ,O>Neill D. Prevention of elder abuse. The Lancet. 2011;377(9782):2005-6. 